\title{
Effects of Combined Upper Limb Robotic Therapy in Patients With Tetraplegic Spinal Cord Injury
}

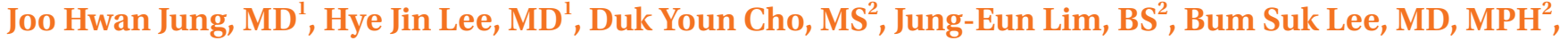 \\ Seung Hyun Kwon, $\mathrm{MD}^{1}$, Hae Young Kim, $\mathrm{MD}^{1}$, Su Jeong Lee, $\mathrm{MD}^{1}$ \\ ${ }^{1}$ Department of Rehabilitation Medicine, National Rehabilitation Center, Seoul; \\ ${ }^{2}$ Translational Research Center for Rehabilitation Robots, National Rehabilitation Center, Seoul, Korea
}

Objective To confirm the effects of combined upper limb robotic therapy (RT) as compared to conventional occupational therapy (OT) in tetraplegic spinal cord injury (SCI) patients and to suggest the optimized treatment guidelines of combined upper limb RT.

Methods After subject recruitment and screening for eligibility, the baseline evaluation for outcome measures were performed. We evaluated the Graded and Redefined Assessment of Strength, Sensibility, and Prehension (GRASSP), the American Spinal Injury Association upper extremity motor score, grip and pinch strength, and the Spinal Cord Independence Measurement III (SCIM-III). In this study, the pre-tested participants were divided randomly into the RT and OT group. The utilized interventions included combined upper limb RT using ArmeoPower and Amadeo (RT group), or conventional OT (OT group) in addition to daily inpatient rehabilitation program. The participants underwent 40 minutes $\times 3$ sessions $\times 5$ weeks of interventions.

Results A total of 30 tetraplegic SCI patients completed entire study program. After 5 weeks of intervention, both groups demonstrated increases in GRASSP-strength and SCIM-III. The manual muscle test scores of elbow flexion, elbow extension, 2-5th metacarpophalangeal extension, and SCIM-III subscores of bathing-upper, dressing-upper, and grooming as well as the GRASSP-qualitative prehension score were noted to have been significantly increased in the RT group as evaluated. The OT group showed improvements in the GRASSP-quantitative prehension score and some items in grip and pinch strength. There was no significant difference between the two groups in almost all measurements except for the SCIM-III bathing-upper subscore.

Conclusion Combined upper limb RT demonstrated beneficial effects on the upper limb motor function in patients with tetraplegic SCI, which were comparable with conventional OT.

Keywords Spinal cord injuries, Upper extremity, Robotics, Rehabilitation

Received December 27, 2018; Accepted February 21, 2019

Corresponding author: Hye Jin Lee

Department of Rehabilitation Medicine, National Rehabilitation Center, 58 Samgaksan-ro, Gangbuk-gu, Seoul 01022, Korea. Tel: +82-2-901-1886, Fax: +82-2-902-3835, E-mail: entreciel80@korea.kr

ORCID: Joo Hwan Jung (http://orcid.org/0000-0003-2329-6812); Hye Jin Lee (http://orcid.org/0000-0001-9400-6283); Duk Youn Cho (http://orcid. org/0000-0002-5170-5030); Jung-Eun Lim (http://orcid.org/0000-0002-5055-0428); Bum Suk Lee (http://orcid.org/0000-0002-0506-0403); Seung Hyun Kwon (http://orcid.org/0000-0001-8700-6306); Hae Young Kim (http://orcid.org/0000-0001-5635-7349); Su Jeong Lee (http://orcid.org/0000-00015794-8167).

(c) This is an open-access article distributed under the terms of the Creative Commons Attribution Non-Commercial License (http://creativecommons.org/ licenses/by-nc/4.0) which permits unrestricted noncommercial use, distribution, and reproduction in any medium, provided the original work is properly cited. Copyright (๑) 2019 by Korean Academy of Rehabilitation Medicine 


\section{INTRODUCTION}

Generally speaking, the annual incidence of spinal cord injury (SCI) ranges from 13.0 to 163.4 cases per million people worldwide. In those cases, the most common level of injury is the cervical spine, which results in patients being tetraplegic [1]. Most tetraplegic SCI patients have limitations in upper limb function leading to dependency in activities of daily living (ADLs), which reduces the quality of life in the case of tetraplegic SCI patients $[2,3]$. Therefore, studies have investigated the effects and feasibility of various treatment options to improve upper limb strength and function in patients. Conventionally, with physiotherapy, occupational therapy (OT) comprises the main components of rehabilitative intervention. OT includes strengthening/endurance exercises, range of motion (ROM)/stretching exercises, and ADL training. Occupational therapists may adopt or modify specific contents of the therapy based on the level of injury, severity of injury, and functional expectations with the goal of facilitating motor recovery of the upper limbs and optimizing functional independence $[4,5]$.

Many studies on which therapy method results in the best outcomes in patients with SCI has been undergone. Neurological recovery in patients with central nervous system (CNS) injury, such as SCI has been rationalized by a theoretical background that rehabilitative therapy improves neuroplasticity of the damaged CNS [6]. Recently, it has been reported that therapy based on the 'motor learning theory' is effective for recovery of the impaired upper limb function. With repetitive movements as close as possible to help achieve the final goal of optimum movement, patients can learn motor skills leading to recovery of the CNS [7]. It has also been suggested that repetitive and activity-based exercise can facilitate recovery after SCI by inducing practice-dependent brain and spinal plasticity [8-10]. Therefore, repetitive, intensive, and activity-based upper limb rehabilitative therapy can be helpful in enhancing impaired upper limb function.

Rehabilitative robotic therapy (RT) provides repetitive and intensive training and can improve the effectiveness of conventional OT or physiotherapy by providing treatment in more accurate, coherent, and detailed way [11]. Various types of upper limb rehabilitative robots have been developed and studied to confirm their feasibility and effectiveness. Several studies involving stroke pa- tients have demonstrated the efficacy and feasibility of upper limb RT $[12,13]$. By the same token, well-designed randomized control trials (RCTs) with a large sample size also showed solid evidence on the efficacy and feasibility of upper limb RT in stroke $[14,15]$.

Meanwhile, it is noted that studies in SCI patients were relatively few when reviewed. Some case series and pilot studies recently showed the feasibility and effect of upper limb RT in SCI patients. In this sense, Zariffa et al. [16] reported a case series on the use of an upper limb rehabilitation device in 12 subacute cervical SCI patients. Cortes et al. [17] performed a single group prospective study on wrist-robot training in 10 adults with chronic tetraplegic SCI. Additionally, Fitle et al. [18] reported a case series on an upper limb robotic exoskeleton in 10 adults with chronic incomplete SCI. However, these studies only demonstrated the feasibility of robots as an objective evaluation tool, rather than the viability of being used as a treatment tool $[16,19-21]$. Furthermore, no study has been shown to have compared more than two combined robotic interventions.

In contrast to conventional OT, where therapists treat the proximal and distal joints together, currently developed upper limb robotics focus on treating only one or two joints. In this context, it would be more effective to combine two robotics to treat proximal and distal part respectively. The aim of this study was to determine the effects of combined upper limb RT in comparison with conventional OT in tetraplegic SCI patients as an adjunctive therapy to conventional OT. In addition, it will be important to show preliminary data that can be helpful in determining indications and treatment protocols of upper limb RT to maximize the advantages of the intervention in SCI patients.

\section{MATERIALS AND METHODS}

\section{Subjects}

We recruited study subjects who were hospitalized in the National Rehabilitation Center, Seoul, Korea from March to December 2017.

The inclusion criteria were an age of 19 years or older, cervical cord injury with a motor level between $\mathrm{C} 2$ and C8, less than 24 months after onset, the American Spinal Injury Association (ASIA) impairment scale (AIS) A to D, enough cognitive ability to understand the contents of 
this study and to follow the directions of the researcher, and patients who decided to participate voluntarily in the study with consent of the patients themselves.

The exclusion criteria were patients with diseases causative of muscle weakness other than SCI (e.g., neuromyopathy), a scale of 3 or more on the Modified Ashworth Scale in upper limbs, which indicated severe spasticity, restrictions to sit on a wheelchair or a chair for $30 \mathrm{~min}$ utes or more, severe shoulder pain, and a limitation of passive ROM by $50 \%$ or more of the normal range in upper limb joints.

General demographic factors of all study subjects were collected and analyzed, which included not only AIS and neurological level of injury, but also sex, age, and duration of injury.

\section{Assessment}

An evaluation was performed before and after intervention in all participants. The primary outcome measure was the Graded and Redefined Assessment of Strength, Sensibility, and Prehension (GRASSP) to confirm overall changes of the intervened upper limb. In this respect, the GRASSP is a valid, reliable, and responsive clinical standardized upper limb impairment measure for acute and chronic cervical SCI [22]. In this measure, the upper limb function is assessed quantitatively through three domains: strength, sensation, and prehension. Details of the assessment tool were described in Supplementary Table S1.

Because of its proven reliability, validity, responsiveness, and sensitivity [22,23], the GRASSP was used in many other studies on upper limb RT in SCI patients,
$[16,21,24,25]$ and is being implemented worldwide for evaluating the efficacy of interventions in a number of investigator driven studies and sponsored clinical trials, and for predicting functional outcome in cervical SCI $[23,26,27]$.

The secondary outcomes measure for this study was ASIA-upper extremity motor score (UEMS), grip and pinch strength of the intervened upper limb, and Spinal Cord Independence Measurement III (SCIM-III) scores.

The UEMS was scored by manual muscle test (MMT) on elbow flexion, wrist extension, elbow extension, 3rd distal interphalangeal flexion, and 5th finger abduction, which are five key muscle movements in C5 to T1. Each score was graded using the medical research council (MRC) scale in accordance with ASIA guidelines [28]. The UEMS of each side range from $0-25$; the side with the lowest baseline UEMS was defined as more affected. Regarding which side to intervene, we selected the more affected side based on initial UEMS.

A hydraulic hand dynamometer (Baseline; Fabrication Enterprises, White Plains, NY, USA) and hydraulic pinch gauge (Baseline; Fabrication Enterprises) were used to measure the grip and pinch strength of the hands and fingers with objective numerical values $(\mathrm{kg})$. The strength was measured three times for full grasp strength, tip pinch strength, lateral pinch strength, and three-jaw chuck pinch strength, and the average value was calculated.

The SCIM-III was used to confirm the change in the independence index in ADLs. The SCIM-III is a valid and reliable outcome measure to address three specific domains for SCI patients which are self-care (six items,
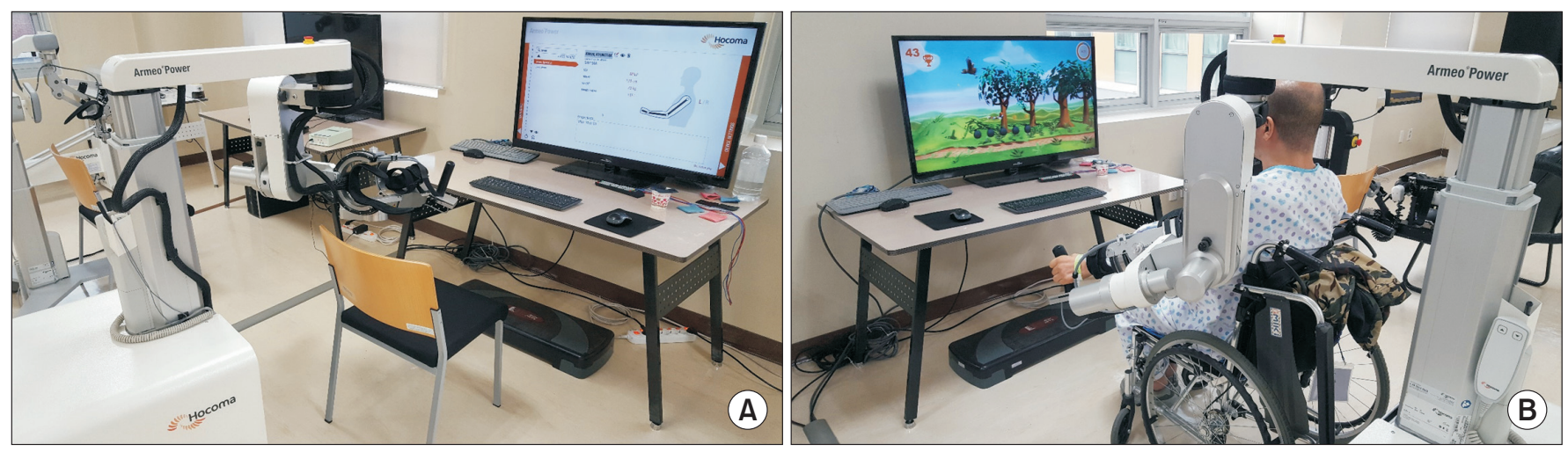

Fig. 1. (A) ArmeoPower, a rehabilitation exoskeleton that mainly trains proximal upper limb including shoulder, elbow, and wrist. (B) The patient training proximal upper limb with ArmeoPower. 
scores 0-20), respiration and sphincter management (four items, scores 0-40), and mobility (nine items, scores 0-40). Total SCIM-III scores range from 0-100; higher scores are indicative of more independence $[29,30]$. We checked only changes in detailed items of the selfcare domain related to upper limbs including feeding, bathing-upper, bathing-lower, dressing-upper, dressinglower, and grooming. The scores of each item range from $0-3$ or $0-4$.

\section{Intervention protocol}

After the pretest, the participants were randomly assigned to the RT group or OT group using block randomisation which was generated using a computer program. We decided to treat the more affected side with lower initial MMT scores, right or left, because the more affected side was thought to have more potential to change after the intervention.

The RT group received, in addition to conventional OT, a combined upper limb RT using both ArmeoPower (Hocoma Inc., Volketswil, Switzerland), which trains the entire upper arm including shoulder, elbow, and wrist and Amadeo (Tyromotion Inc., Graz, Austria), which trains the hand. Training was proceeded in a proximal-to-distal sequence: ArmeoPower 20 minutes and Amadeo 20 minutes. ArmeoPower can induce or assist the movement of the patient by expanding or narrowing the ROM according to the patient's therapeutic purpose (Fig. 1). This robot includes 21 games, which can be categorized according to therapeutic purposes and joints. Each game can be adjusted by difficulty level (3 steps), progress time (2-5 min), and degree of arm support (0\%-100\%). Amadeo is a device that can rehabilitate the fingers and hands of the distal upper limb motor dysfunction patients (Fig. 2). Amadeo moves the patient's fingers according to the software defined pattern. The treatment program includes continuous passive motion (CPM), CPM plus, assistive therapy, motility, memory, balloon, and fire fighters. The CPM provides training for 'simultaneous movement of thumb and fingers' and 'crossing movement of thumb and fingers.' In CPM plus, as extended version of CPM, biofeedback is offered in addition. Biofeedback was offered by expressing the recorded finger's power during flexion and extension exercises in the form of a smile, thereby helping motivate the patient to focus on their motion. In assistive therapy, the robot will help the pa- tient to do the flexion and extension actions to the end, when the side of the finger the patient is moving no longer moves. Other Amadeo programs provide training for finger flexion and extension. In RT, the therapist choses different programs with individualized settings to train specific areas where patients had prior difficulties in regards to movements or to strengthen relatively weak upper limb muscles.

The OT group received conventional OT in a proximalto-distal treatment sequence in a manner similar to the RT group by well trained, experienced occupational therapists. The therapy was individualized to patients and focused on relatively weak muscles and clumsy movement based on the pretest. Task-oriented strengthening and fine motor training were included in the therapy, but the ADL training was not included. For some patients, visual feedback with a mirror was provided during the timeframe of the repetition of movement. During conventional OT, the therapist provided minimal assistance to induce voluntary movement in the participant as much as possible. When training the proximal upper limb, the therapist instructed patients to hold something or make a fist for simultaneous volition of the distal muscles.

The participants performed a total of 15 intervention sessions at an intensity of 40 minutes per session, 1 session per day, 3 days a week, for 5 weeks in both groups. The intervention ran parallel to the daily inpatient rehabilitation program, which included 30 minutes conven-
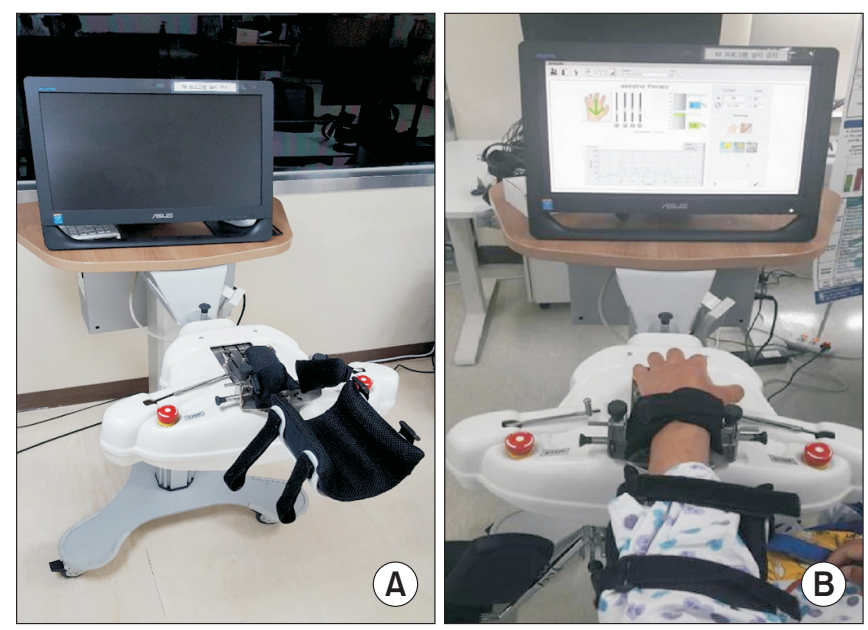

Fig. 2. (A) Amadeo, a rehabilitation exoskeleton that trains distal upper limb including hand and fingers. (B) The patient training distal upper limb with Amadeo. 
tional OT once daily. Before and after the intervention, an evaluation was performed in all of the participants.

\section{IRB approval}

In March 2017, we underwent approval for this study at the first regular Institutional Review Board (IRB) review conducted in the National Rehabilitation Center (No. NRC-2017-01-002). The study period was 1 year after deliberation from March 8, 2017 to March 7, 2018.

\section{Statistical analyses}

Descriptive statistical analysis was performed for the general demographics of the study subjects. For an evaluation of the noted changes in the outcome measures before and after intervention, the Wilcoxon signed-rank test was employed to compare scores of GRASSP strength, sensation, and prehension, UEMS, grip/pinch strength, and SCIM-III. The Kruskal-Wallis test was employed to compare between the two groups. All statistical analysis was conducted by the Korean version of Windows SPSS 20.0 (IBM SPSS, Armonk, NY, USA). The level of statistical significance was defined as $\mathrm{p}<0.05$.

\section{RESULTS}

The patients were recruited after IRB approval in March
2017. A total of 43 patients were screened by December 2017, but 5 patients were excluded due to aggravation of a pressure sore, refusal to participate for personal reasons, shoulder pain, and declined medical condition. Thirty-eight of 43 patients were evaluated in advance and randomly assigned into the RT group $(\mathrm{n}=22)$ or OT group $(\mathrm{n}=16)$. Five patients in the RT group and three patients in the OT group dropped out due to early discharge, refusal to participate in the middle of the intervention, and declined medical condition. Finally, 17 patients in the RT group (14 men; mean age, 47 years; mean post-SCI, 7 months) and 13 patients in the OT group (10 men; mean age, 53 years; mean post-SCI, 7 months) completed all intervention sessions and underwent the post-test (Fig. 3). In both groups, AIS-D was noted as the most frequent AIS. Additionally, the general demographics of the two groups are demonstrated in Table 1.

\section{Primary outcome measure (GRASSP)}

There were statistically significant increases in the total GRASSP-strength scores in both groups with different results in each muscle. The RT group showed significant improvements in MMT scores of shoulder abduction, elbow flexion, elbow extension, and 2-5th metacarpophalangeal (MP) extension. On the other hand, the OT group showed significant improvements in shoulder abduction,

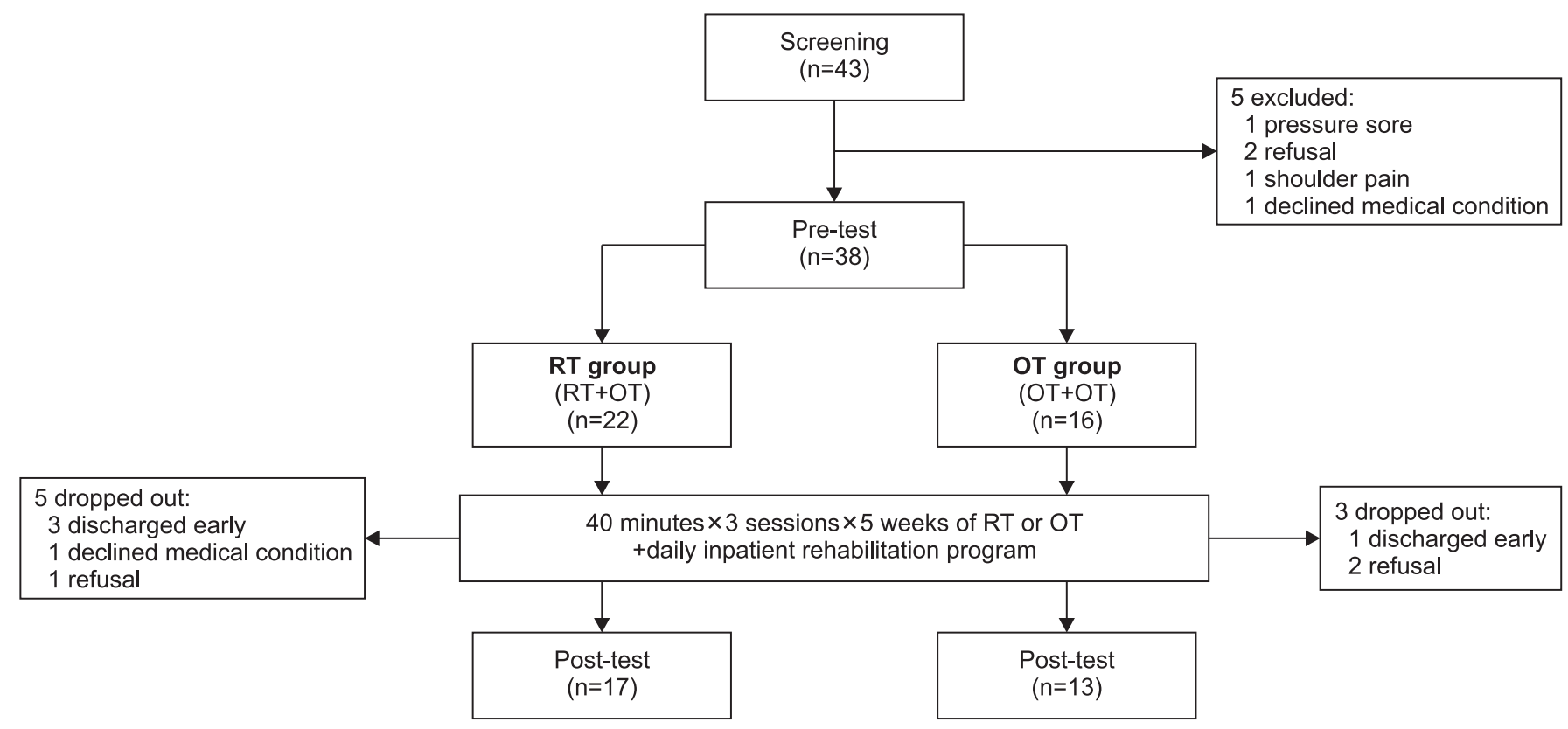

Fig. 3. Flow chart of subject recruitment. RT, combining upper limb robotic therapy with conventional occupational therapy; OT, only conventional occupational therapy. 
Table 1. General demographics of the study subjects

\begin{tabular}{lccc}
\hline Demographic factors & RT $(\mathbf{n}=\mathbf{1 7})$ & OT $(\mathbf{n}=\mathbf{1 3})$ & $\mathbf{p}$-value \\
\hline Sex & & & 1.000 \\
$\quad$ Male & 14 & 10 & \\
$\quad$ Female & 3 & 3 & \\
Age (yr) & $47.23 \pm 14.01$ & $53.00 \pm 13.50$ & 0.198 \\
$\begin{array}{l}\text { Disease duration (mo) } \\
\text { AIS grade }\end{array}$ & $7.53 \pm 6.51$ & $7.62 \pm 5.22$ & 0.773 \\
\hline AIS-A & & & \\
AIS-B & 3 & 0 & \\
AIS-C & 2 & 2 & \\
AIS-D & 4 & 3 & \\
Neurological level & 8 & 8 & \\
of injury & & & \\
C2 & & & \\
\hline C3 & 0 & 1 & \\
C4 & 1 & 5 & \\
\hline C5 & 5 & 4 & \\
\hline C6 & 4 & 0 & \\
\hline C7 & 3 & 2 & \\
\hline C8 & 4 & 1 & \\
\hline
\end{tabular}

Values are presented as mean \pm standard deviation.

RT, combining upper limb robotic therapy with conventional occupational therapy; OT, only conventional occupational therapy; AIS, American Spinal Injury Association impairment scale. wrist extension, and 5th finger abduction (Table 2). There was no significant difference between the two groups.

There were also different results in the changes of function of the intervened upper limb as measured by the prehension domain in GRASSP. The RT group had significant improvements in cylindrical grasp, lateral key pinch, and total in qualitative prehension, as well as 'pick up four coins \& place in slot' in quantitative prehension. Whereas the OT group had significant improvements in total score of quantitative prehension after the intervention. When comparing the two groups, no significant difference was observed (Table 3).

In GRASSP-sensation, there was no significant change after the intervention.

\section{Secondary outcome measures}

The UEMSs of both groups were increased significantly after intervention, which were the same as the changes in the GRASSP-strength assessment (Supplementary Table S2).

In the meantime, the RT group had no significant improvements in grip strength measured by a hand-held dynamometer. On the other hand, the OT group showed significant improvement in grip strength of the tip pinch and three-jaw chuck items (Table 4). There was no sig-

Table 2. Changes in GRASSP-strength scores after intervention

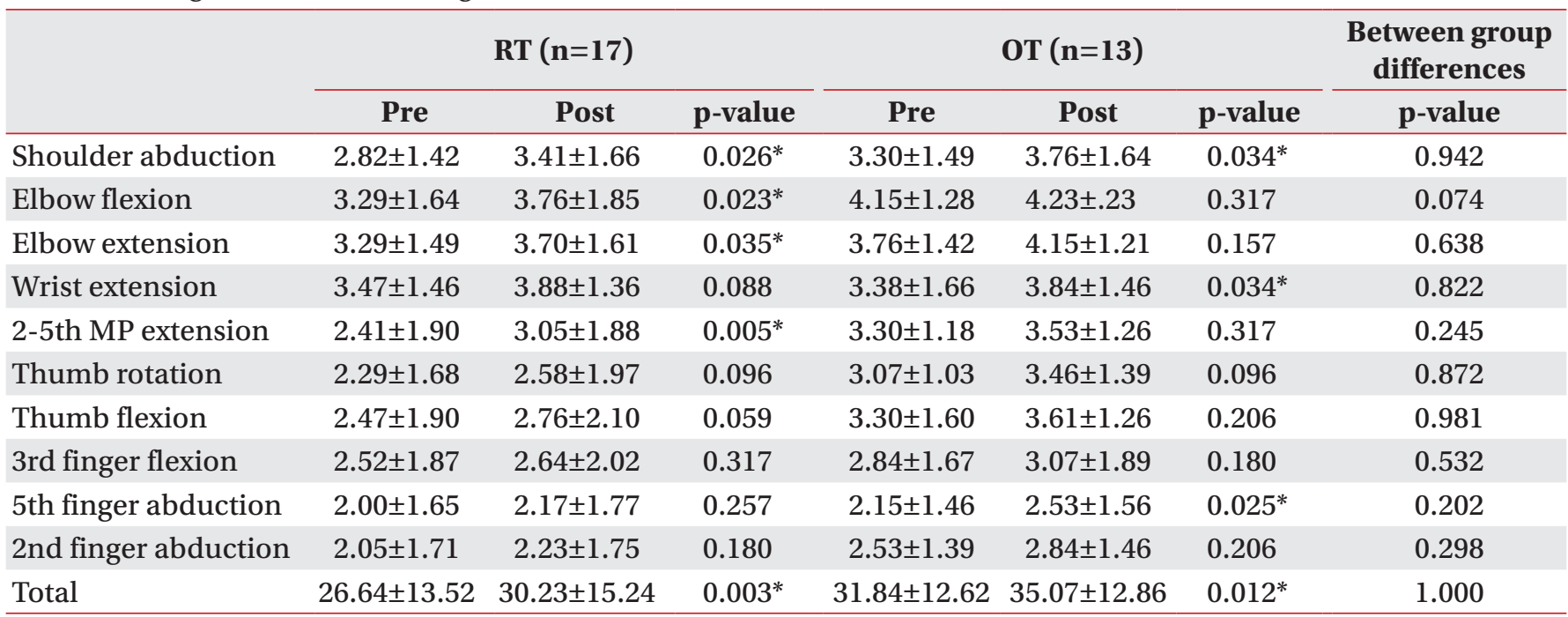

Values are presented as mean \pm standard deviation.

GRASSP, Graded and Redefined Assessment of Strength, Sensibility and Prehension; RT, combining upper limb robotic therapy with conventional occupational therapy; OT, only conventional occupational therapy; MP, metacarpophalangeal.

${ }^{*} \mathrm{p}<0.05$ by Wilcoxon signed-rank test. 
Table 3. Changes in GRASSP-prehension scores after intervention

\begin{tabular}{|c|c|c|c|c|c|c|c|}
\hline & \multicolumn{3}{|c|}{$\operatorname{RT}(n=17)$} & \multicolumn{3}{|c|}{ OT $(n=13)$} & \multirow{2}{*}{$\begin{array}{c}\begin{array}{c}\text { Between group } \\
\text { differences }\end{array} \\
\text { p-value }\end{array}$} \\
\hline & Pre & Post & p-value & Pre & Post & p-value & \\
\hline Cylindrical grasp & $1.88 \pm 1.65$ & $2.17 \pm 1.74$ & $0.025^{*}$ & $2.23 \pm 1.53$ & $2.46 \pm 1.50$ & 0.670 & 0.888 \\
\hline Lateral key pinch & $2.05 \pm 1.71$ & $2.35 \pm 1.93$ & $0.025^{*}$ & $2.38 \pm 1.38$ & $2.46 \pm 1.61$ & 0.943 & 0.415 \\
\hline Tip to tip pinch & $2.29 \pm 1.89$ & $2.35 \pm 1.93$ & 0.317 & $2.61 \pm 1.66$ & $2.69 \pm 1.60$ & 0.916 & 0.821 \\
\hline Qualitative total & $6.23 \pm 5.14$ & $6.88 \pm 5.54$ & $0.015^{*}$ & $7.23 \pm 4.41$ & $7.61 \pm 4.62$ & 0.833 & 0.653 \\
\hline Poor water from a bottle & $2.47 \pm 2.18$ & $2.35 \pm 2.31$ & 0.705 & $2.69 \pm 2.13$ & $3.30 \pm 2.01$ & 0.063 & 0.085 \\
\hline Open jars & $2.47 \pm 2.06$ & $2.76 \pm 1.92$ & 0.180 & $3.15 \pm 1.95$ & $3.30 \pm 2.01$ & 0.157 & 0.887 \\
\hline $\begin{array}{l}\text { Transfer } 9 \text { pegs board } \\
\text { to board }\end{array}$ & $2.88 \pm 2.02$ & $2.88 \pm 2.02$ & 1.000 & $3.15 \pm 1.95$ & $3.23 \pm 2.08$ & 0.655 & 0.697 \\
\hline Pick up \& turn a key & $2.23 \pm 2.01$ & $2.47 \pm 2.00$ & 0.257 & $2.84 \pm 2.15$ & $3.15 \pm 1.95$ & 0.257 & 0.829 \\
\hline $\begin{array}{l}\text { Pick up } 4 \text { coins \& place } \\
\text { in slot }\end{array}$ & $2.23 \pm 2.10$ & $2.70 \pm 2.14$ & $0.021^{*}$ & $2.84 \pm 1.90$ & $3.07 \pm 1.97$ & 0.180 & 0.193 \\
\hline Screw 4 nuts onto bolts & $1.17 \pm 1.59$ & $1.41 \pm 1.58$ & 0.194 & $1.92 \pm 1.80$ & $2.30 \pm 1.88$ & 0.102 & 0.534 \\
\hline Quantitative total & $13.47 \pm 11.27$ & $14.58 \pm 11.39$ & 0.107 & $16.61 \pm 11.30$ & $18.38 \pm 11.57$ & $0.027^{*}$ & 0.861 \\
\hline
\end{tabular}

Values are presented as mean \pm standard deviation.

GRASSP, Graded and Redefined Assessment of Strength, Sensibility and Prehension; RT, combining upper limb robotic therapy with conventional occupational therapy; OT, only conventional occupational therapy.

${ }^{*} \mathrm{p}<0.05$ by Wilcoxon signed-rank test.

Table 4. Changes in grip and pinch strength $(\mathrm{kg})$ after intervention

\begin{tabular}{|c|c|c|c|c|c|c|c|}
\hline & \multicolumn{3}{|c|}{ RT $(n=17)$} & \multicolumn{3}{|c|}{ OT $(n=13)$} & \multirow{2}{*}{$\begin{array}{c}\begin{array}{c}\text { Between group } \\
\text { differences }\end{array} \\
\text { p-value } \\
\end{array}$} \\
\hline & Pre & Post & p-value & Pre & Post & p-value & \\
\hline Full grasp & $4.26 \pm 4.54$ & $5.26 \pm 5.84$ & 0.213 & $7.14 \pm 7.27$ & $8.50 \pm 7.45$ & 0.139 & 0.506 \\
\hline Tip pinch & $0.91 \pm 0.97$ & $0.88 \pm 1.02$ & 0.798 & $1.36 \pm 1.32$ & $1.75 \pm 1.67$ & $0.044^{*}$ & 0.212 \\
\hline Lateral pinch & $2.28 \pm 2.32$ & $2.38 \pm 2.33$ & 0.878 & $2.66 \pm 2.04$ & $3.23 \pm 2.76$ & 0.107 & 0.098 \\
\hline Three-jaw chuck & $1.52 \pm 1.52$ & $1.55 \pm 1.53$ & 0.624 & $1.50 \pm 1.63$ & $2.47 \pm 2.36$ & $0.030^{*}$ & 0.062 \\
\hline
\end{tabular}

Values are presented as mean \pm standard deviation.

RT, combining upper limb robotic therapy with conventional occupational therapy; OT, only conventional occupational therapy.

${ }^{*} \mathrm{p}<0.05$ by Wilcoxon signed-rank test.

nificant difference in UEMS and grip/pinch strength between the two groups.

In SCIM-III, the RT group showed significant improvements in three items (bathing-upper, dressing-upper, and grooming), whereas the OT group showed improvements in only one item (dressing-lower). It is noted that both groups showed significant increase in total score of SCIM-III. There was a significant difference in one item score (bathing-upper) between the two groups (Table 5).

\section{DISCUSSION}

This study aimed to investigate the effects of combined upper limb RT in comparison with conventional OT in patients with subacute tetraplegic SCI, and to develop a protocol based on these results. After 5 weeks of intervention, both groups demonstrated improvements in strength and function of the intervened upper limb measured by MMT, grip strength, SCIM-III, and GRASSP as compared to before the intervention.

Recently, there have been enhanced efforts to restore 
Table 5. Changes in SCIM-III scores after intervention

\begin{tabular}{|c|c|c|c|c|c|c|c|}
\hline & \multicolumn{3}{|c|}{ RT $(n=17)$} & \multicolumn{3}{|c|}{ OT $(n=13)$} & \multirow{2}{*}{$\begin{array}{c}\begin{array}{c}\text { Between group } \\
\text { differences }\end{array} \\
\text { p-value }\end{array}$} \\
\hline & Pre & Post & p-value & Pre & Post & p-value & \\
\hline Feeding & $1.52 \pm 1.00$ & $1.82 \pm 1.13$ & 0.059 & $1.84 \pm 1.06$ & $1.84 \pm 1.14$ & 1.000 & 0.214 \\
\hline Bathing-upper & $0.52 \pm 0.87$ & $1.00 \pm 1.00$ & $0.011^{*}$ & $0.84 \pm 1.14$ & $0.92 \pm 1.32$ & 0.655 & $0.043^{* *}$ \\
\hline Bathing-lower & $0.41 \pm 0.79$ & $0.64 \pm 1.05$ & 0.234 & $0.38 \pm 0.96$ & $0.53 \pm 1.05$ & 0.317 & 0.799 \\
\hline Dressing-upper & $1.00 \pm 1.41$ & $1.70 \pm 1.72$ & $0.028^{*}$ & $1.23 \pm 1.58$ & $1.92 \pm 1.75$ & 0.071 & 0.909 \\
\hline Dressing-lower & $0.94 \pm 1.19$ & $1.29 \pm 1.64$ & 0.107 & $0.53 \pm 1.05$ & $1.07 \pm 1.44$ & $0.034^{*}$ & 0.623 \\
\hline Grooming & $1.47 \pm 1.17$ & $1.76 \pm 1.20$ & $0.025^{*}$ & $1.84 \pm 1.28$ & $1.92 \pm 1.75$ & 0.317 & 0.147 \\
\hline Total & $5.88 \pm 5.34$ & $8.23 \pm 6.74$ & $0.008^{*}$ & $6.69 \pm 5.80$ & $8.23 \pm 6.78$ & $0.024^{*}$ & 0.466 \\
\hline
\end{tabular}

Values are presented as mean \pm standard deviation.

SCIM-III, Spinal Cord Independence Measurement III; RT, combining upper limb robotic therapy with conventional occupational therapy; OT, only conventional occupational therapy.

${ }^{*} \mathrm{p}<0.05$ by Wilcoxon signed-rank test.

${ }^{* *} \mathrm{p}<0.05$ by Kruskal-Wallis test.

upper limb function in quadriplegia patients due to CNS injury, such as a stroke or SCI. These efforts include various types of exercise therapy, tendon/nerve transfer surgery, electrical stimulation, functional electrical stimulation, upper limb RT, and repetitive transcranial magnetic stimulation [31-35]. Among these, upper limb RT is emerging as a promising therapy in CNS injury patients, chiefly because of its advantage in intensive repetitive training.

In this sense, it is noted that several types of upper limb rehabilitative robots have been developed for use with patients, and there are 6-7 types that are currently available. Studies on the effectiveness and feasibility of these robots have been actively carried out as have been applied for use in brain lesioned patients [12-15,36,37]. Many studies have shown good results on the effectiveness of RT in stroke patients. Klamroth-Marganska et al. [15] performed a study on an exoskeletal upper limb RT in chronic patients with stroke; the improvement in upper limb function evaluated by the Fugl-Meyer Assessment (FMA-UE) in the robot-treated group was statistically larger than the improvements as noted in the conventional treatment group. The authors suggested that robots can provide high-intensity training, and a patient cooperative control strategy enabling specific tasks to be performed by the patient's voluntary movement can be of benefit in these cases.

In the RCT on robot-assisted hand therapy by Sale et al. [13], participants with acute stroke showed improve- ments in FMA-UE, Box and Block Test, Motricity Index, and MRC scales after the use of an upper limb RT; highintensity training was indicated as a major factor in upper limb function recovery. Also, in other RCT by Takahashi et al. [14], the hemiplegic participants received upper limb therapy using a stationary fixed-based, end-effector arm rehabilitative robot in addition to the use of conventional OT. When compared to the control group that received conventional OT alone, the experimental group demonstrated improvements in flexor synergy and proximal upper extremity scores of FMA-UE.

In this study, we combined two robots, which covered the training of the proximal and distal upper limbs. With ArmeoPower, we trained shoulder and elbow movement with or without assistance which could be adjustable to each patient's level. Amadeo could train wrist and finger movement with or without assistance. Passive ROM exercise was possible with both robots in patients with little or no motor power in corresponding part.

Combined RT in stoke patients was previously reported by Hesse et al. [38]. In this study, four different endeffector type robots were selected; two robots to train the shoulder and elbow, one to train the wrist and one to train the fingers. Significant improvements in the FMA-UE scores were demonstrated in participants who received RT plus individual arm therapy, which corresponds with the results of conventional OT in our study.

On the other hand, studies on upper limb RT in SCI patients are still insufficient with controversial results. Also, 
there are few RCTs comparing RT with conventional OT. Most studies were performed in chronic patients, and studies that applied more than two combined robotics are rare. However, there are several studies that showed positive effects of upper limb RT in SCI. In a case report by Lu et al. [25], a patient with chronic incomplete tetraplegic SCI showed improvement in grip/pinch strength, Box and Block Test, and GRASSP score after hand training using a rehabilitative robot. The authors described that real-time feedback on the patient's own motor intention during the RT helped the CNS to map motion intentions to the desired finger movements. A study by Francisco et al. [11] showed improved upper limb function as evaluated by arm and hand function tests (Jebsen-Taylor Hand Function Test, Action Research Arm Test), strength of upper limb (UEMS, grip/pinch strength), and independence in ADLs (SCIM-II) after RT in chronic tetraplegic SCI patients. Highly-repetitive training of the upper limb by the robot was thought to be critical in the improvement of strength of the muscles. Cortes et al. [17] also reported improvements in motor kinematics (aim, smoothness) after upper limb RT in chronic tetraplegic SCI patients.

The present findings correspond well with prior experimental studies. When looking in detail, the RT group showed a significant improvement in MMT scores of the proximal upper limb muscles (shoulder abduction, elbow flexion, and elbow extension) and the SCIM-III scores in items related to upper limb function (bathing-upper, dressing-upper, and grooming). In contrast, changes in the MMT scores of the proximal upper limb muscles in the OT group were not distinct, and neither were those of the SCIM-III scores, except for the dressing-lower. Thus, we could confirm the different effects of the intervention between the two groups in the proximal upper limb. It has been mentioned in previous studies on RT that repetitive and intensive therapy using robotics is effective in muscle strengthening. In this study, repetitive and intensive proximal muscle training by Armeo was thought to be very effective in the strengthening of proximal muscles. Furthermore, improvement in proximal muscles strength can be attributed to the noted improvement in the ADLs evaluated by SCIM-III, as the items mainly use the proximal upper limb rather than the distal part.

Changes in the distal upper limb were also noted as different between the two groups. The RT group showed significant improvements in two items of the GRASSP-quali- tative prehension subscores (cylindrical grasp, lateral key pinch), as well as the total score which evaluate voluntary control of wrist and digits when generating three different grasps. On the other hand, there was no significant changes in the GRASSP-quantitative prehension scores, which evaluate distal fine motor function with taskspecific items. This is different from the change seen in the OT group, which showed a significant increase in the total GRASSP-quantitative prehension score. This result is in close agreement with a previous study by OrihuelaEspina et al. [39]. In the study, after robot training for hand motor recovery using Amadeo in subacute stroke patients, improvements in FMA hand and the Motricity Index were demonstrated. The Amadeo program was thought to be effective in qualitative prehension training by enabling the repetitive training of hand flexion and extension with high-intensity in patients with impaired hand function at the target of relatively simple prehensive motion in our study. In contrast to the fact that at the therapist's discretion, which allows patients to freely practice complex tasks in a three-dimensional space, movements in the robot settings are still limited in designated axes and planes with simple motion which can explain the result of the evaluation. Meanwhile, as in the stroke population, spinal cord motor circuits undergo physiological reorganization after SCI, which results in delayed functional recovery in patients with SCI for several years after injury. Somatosensory stimulation promotes sensorimotor cortical plasticity leading to changes in motor performance [40]. In the same context, we concluded that tactile sensory stimulation and proprioception feedback during conventional OT might have contributed to restoring some of the motor control functions of the distal upper limb in the OT group.

Differences in the distal muscle strength were also noted. There was no significant increase in MMT scores of distal muscles except for 2-5th MP extension and grip/ pinch strength in the RT group, suggesting that the RT may be somewhat lacking in improving finger strength. Currently, Amadeo is able to train individual movements of fingers only in the motility mode. In most game programs, support was not provided so that patients with weak distal muscles had difficulties with utilizing this robotics. This is thought to be an important reason why the improvement in finger strength was not significant. The OT group demonstrated improvements in MMT 
scores of wrist extension, 5th finger abduction, grip/ pinch strength measured by dynamometer (tip pinch, and three-jaw chuck). During conventional OT, the proximal muscle was not just simply moved up and down and left and right. In addition to these movements, the upper limb was moved with training of the distal muscle such as simultaneously grabbing an object like a ball. But when training distal muscles, the upper limb was placed on the desk and patients were required to focus on only the distal motion. Therefore, simultaneous training of the distal part while proximal training increased the total dose of distal training, which may have been effective in strengthening of the distal muscles.

The aforementioned results suggest that combined upper limb RT is effective for proximal strengthening and training of simple prehensive motion of the impaired upper limb, whereas conventional OT is noted to be effective for the distal strengthening and distal fine motor function.

All evaluations in the study did not show a significant difference when comparing the RT and OT group, but there were significant differences in the items with significant improvement, suggesting that they have different therapeutic effects. By combining these results, it is recommended to apply RT in proximal muscle strengthening, its associated ADLs, and training for ability to generate grasps. However, if distal fine motor function and distal muscle strengthening associated with performing a task is more important, it is conceivable that the conventional OT can be more helpful and appropriate treatment option as compared to RT.

The study has some limitations. First, the study was performed in a single center with availability of only specific types of upper limb rehabilitative robots. Second, there was a difference between the RT and OT group in general characteristics that would affect the outcome such as AIS, level of injury, and baseline outcome results, which was not adjusted in the data for this study due to the small sample size. Third, acute or subacute SCI patients were included in the study, and it was difficult to differentiate changes by RT from those of natural neurological recovery. Fourth, there were consistent principles within the interventions (RT and OT), but customizing therapy for each participant resulted in heterogeneity with the difference in number of repetitions, and the presence and type of feedback, which had confounding potentials and data information.

Conventional OT has been recognized as an important treatment for the recovery of upper limb function in SCI patients. In this study, combined upper limb RT was proven to be effective in enhancing the strength and function of both the distal and proximal part of an upper limb in SCI patients, with relative advantages in developing proximal strengthening in comparison with OT. Unlike RT, conventional OT showed a considerable effect on the improvement of fine motor function of the distal upper limb. In conclusion, by appropriately increasing or decreasing doses of each treatment options according to the patient's condition, a combined upper limb RT and conventional OT can optimize the treatment effect to enhance recovery of upper limb function in patients with tetraplegic SCI.

\section{CONFLICT OF INTEREST}

No potential conflict of interest relevant to this article was reported.

\section{ACKNOWLEDGMENTS}

This study was supported by a grant (No. NRCTRIN17001) of the Translational Research Center for Rehabilitation Robots, Korea National Rehabilitation Center, Ministry of Health \& Welfare, Korea.

\section{AUTHOR CONTRIBUTION}

Conceptualization: Lee HJ, Jung JH, Cho DY, Lim JE. Methodology: Lee HJ, Jung JH, Cho DY, Lim JE. Formal analysis: Lee HJ, Jung JH, Cho DY, Lim JE. Funding acquisition: Lee HJ, Lee BS. Project administration: Jung JH, Cho DY, Lim JE, Kwon SH, Kim HY, Lee SJ. Visualization: Jung JH, Lim JE. Writing original draft: Jung JH, Lee HJ. Writing review and editing: Jung JH, Lee HJ. Approval of final manuscript: all authors.

\section{SUPPLEMENTARY MATERIALS}

Supplementary materials can be found via https://doi. org/10.5535/arm.2019.43.4.445. Table S1. The detailed methods of the Graded and Redefined Assessment of Strength, Sensibility, and Prehension (GRASSP). Table S2. 
Changes in MMT scores of key muscles and UEMSs after intervention.

\section{REFERENCES}

1. Kang Y, Ding H, Zhou H, Wei Z, Liu L, Pan D, et al. Epidemiology of worldwide spinal cord injury: a literature review. J Neurorestoratol 2017;6:1-9.

2. Anderson KD. Targeting recovery: priorities of the spinal cord-injured population. J Neurotrauma 2004;21:1371-83.

3. Snoek GJ, IJzerman MJ, Post MW, Stiggelbout AM, Roach MJ, Zilvold G. Choice-based evaluation for the improvement of upper-extremity function compared with other impairments in tetraplegia. Arch Phys Med Rehabil 2005;86:1623-30.

4. Foy T, Perritt G, Thimmaiah D, Heisler L, Offutt JL, Cantoni K, et al. The SCIRehab project: treatment time spent in SCI rehabilitation: occupational therapy treatment time during inpatient spinal cord injury rehabilitation. J Spinal Cord Med 2011;34:162-75.

5. Ozelie R, Gassaway J, Buchman E, Thimmaiah D, Heisler L, Cantoni K, et al. Relationship of occupational therapy inpatient rehabilitation interventions and patient characteristics to outcomes following spinal cord injury: the SCIRehab project. J Spinal Cord Med 2012;35:527-46.

6. Behrman AL, Bowden MG, Nair PM. Neuroplasticity after spinal cord injury and training: an emerging paradigm shift in rehabilitation and walking recovery. Phys Ther 2006;86:1406-25.

7. Cano-de-la-Cuerda R, Molero-Sanchez A, CarratalaTejada M, Alguacil-Diego IM, Molina-Rueda F, Miangolarra-Page JC, et al. Theories and control models and motor learning: clinical applications in neurorehabilitation. Neurologia 2015;30:32-41.

8. Lynskey JV, Belanger A, Jung R. Activity-dependent plasticity in spinal cord injury. J Rehabil Res Dev 2008;45:229-40.

9. Onifer SM, Smith GM, Fouad K. Plasticity after spinal cord injury: relevance to recovery and approaches to facilitate it. Neurotherapeutics 2011;8:283-93.

10. Quel de Oliveira C, Refshauge K, Middleton J, de Jong L, Davis GM. Effects of activity-based therapy interventions on mobility, independence, and quality of life for people with spinal cord injuries: a sys- tematic review and meta-analysis. J Neurotrauma 2017;34:1726-43.

11. Francisco GE, Yozbatiran N, Berliner J, O'Malley MK, Pehlivan AU, Kadivar Z, et al. Robot-assisted training of arm and hand movement shows functional improvements for incomplete cervical spinal cord injury. Am J Phys Med Rehabil 2017;96(10 Suppl 1):S171S177.

12. Godfrey SB, Holley RJ, Lum PS. Clinical effects of using HEXORR (Hand Exoskeleton Rehabilitation Robot) for movement therapy in stroke rehabilitation. Am J Phys Med Rehabil 2013;92:947-58.

13. Sale P, Mazzoleni S, Lombardi V, Galafate D, Massimiani MP, Posteraro F, et al. Recovery of hand function with robot-assisted therapy in acute stroke patients: a randomized-controlled trial. Int J Rehabil Res 2014;37:236-42.

14. Takahashi K, Domen K, Sakamoto T, Toshima M, Otaka Y, Seto M, et al. Efficacy of upper extremity robotic therapy in subacute poststroke hemiplegia: an exploratory randomized trial. Stroke 2016;47:1385-8.

15. Klamroth-Marganska V, Blanco J, Campen K, Curt A, Dietz V, Ettlin T, et al. Three-dimensional, task-specific robot therapy of the arm after stroke: a multicentre, parallel-group randomised trial. Lancet Neurol 2014;13:159-66.

16.Zariffa J, Kapadia N, Kramer JL, Taylor P, AlizadehMeghrazi M, Zivanovic V, et al. Feasibility and efficacy of upper limb robotic rehabilitation in a subacute cervical spinal cord injury population. Spinal Cord 2012;50:220-6.

17. Cortes M, Elder J, Rykman A, Murray L, Avedissian $\mathrm{M}$, Stampas A, et al. Improved motor performance in chronic spinal cord injury following upper-limb robotic training. NeuroRehabilitation 2013;33:57-65.

18. Fitle KD, Pehlivan AU, O'Malley MK. A robotic exoskeleton for rehabilitation and assessment of the upper limb following incomplete spinal cord injury. Proceedings of 2015 IEEE International Conference on Robotics and Automation (ICRA); 2015 May 26-30; Seattle, WA. p. 4960-6.

19. Kadivar Z, Sullivan JL, Eng DP, Pehlivan AU, O'Malley MK, Yozbatiran N, et al. Robotic training and kinematic analysis of arm and hand after incomplete spinal cord injury: a case study. IEEE Int Conf Rehabil Robot 2011;2011:5975429. 
20.Zariffa J, Kapadia N, Kramer JL, Taylor P, AlizadehMeghrazi M, Zivanovic V, et al. Relationship between clinical assessments of function and measurements from an upper-limb robotic rehabilitation device in cervical spinal cord injury. IEEE Trans Neural Syst Rehabil Eng 2012;20:341-50.

21. Zariffa J, Kapadia N, Kramer JL, Taylor P, AlizadehMeghrazi M, Zivanovic V, et al. Effect of a robotic rehabilitation device on upper limb function in a subacute cervical spinal cord injury population. IEEE Int Conf Rehabil Robot 2011;2011:5975400.

22. Kalsi-Ryan S, Beaton D, Curt A, Duff S, Popovic MR, Rudhe C, et al. The Graded Redefined Assessment of Strength Sensibility and Prehension: reliability and validity. J Neurotrauma 2012;29:905-14.

23. Kalsi-Ryan S, Beaton D, Ahn H, Askes H, Drew B, Curt A, et al. Responsiveness, sensitivity, and minimally detectable difference of the Graded and Redefined Assessment of Strength, Sensibility, and Prehension, Version 1.0. J Neurotrauma 2016;33:307-14.

24. Frullo JM, Elinger J, Pehlivan AU, Fitle K, Nedley K, Francisco GE, et al. Effects of assist-as-needed upper extremity robotic therapy after incomplete spinal cord injury: a parallel-group controlled trial. Front Neurorobot 2017;11:26.

25. Lu Z, Tong KY, Shin H, Stampas A, Zhou P. Robotic hand-assisted training for spinal cord injury driven by myoelectric pattern recognition: a case report. Am J Phys Med Rehabil 2017;96(10 Suppl 1):S146-S149.

26. Steeves JD, Lammertse DP, Kramer JL, Kleitman N, Kalsi-Ryan S, Jones L, et al. Outcome measures for acute/subacute cervical sensorimotor complete (AISA) spinal cord injury during a phase 2 clinical trial. Top Spinal Cord Inj Rehabil 2012;18:1-14.

27.Zariffa J, Curt A, Verrier MC, Fehlings MG, Kalsi-Ryan S; GRASSP Cross-Sectional Study Team and Ontario GRASSP Longitudinal Study Team. Predicting task performance from upper extremity impairment measures after cervical spinal cord injury. Spinal Cord 2016;54:1145-51.

28. Steeves JD, Lammertse D, Curt A, Fawcett JW, Tuszynski $\mathrm{MH}$, Ditunno JF, et al. Guidelines for the conduct of clinical trials for spinal cord injury (SCI) as developed by the ICCP panel: clinical trial outcome measures. Spinal Cord 2007;45:206-21.

29. Anderson K, Aito S, Atkins M, Biering-Sorensen F,
Charlifue S, Curt A, et al. Functional recovery measures for spinal cord injury: an evidence-based review for clinical practice and research. J Spinal Cord Med 2008;31:133-44.

30. Itzkovich M, Gelernter I, Biering-Sorensen F, Weeks C, Laramee MT, Craven BC, et al. The Spinal Cord Independence Measure (SCIM) version III: reliability and validity in a multi-center international study. Disabil Rehabil 2007;29:1926-33.

31. Lu X, Battistuzzo CR, Zoghi M, Galea MP. Effects of training on upper limb function after cervical spinal cord injury: a systematic review. Clin Rehabil 2015;29:3-13.

32. Kloosterman MG, Snoek GJ, Jannink MJ. Systematic review of the effects of exercise therapy on the upper extremity of patients with spinal-cord injury. Spinal Cord 2009;47:196-203.

33. Dunn JA, Sinnott KA, Rothwell AG, Mohammed KD, Simcock JW. Tendon transfer surgery for people with tetraplegia: an overview. Arch Phys Med Rehabil 2016;97(6 Suppl):S75-80.

34. Fox IK, Novak CB, Krauss EM, Hoben GM, Zaidman $\mathrm{CM}$, Ruvinskaya $\mathrm{R}$, et al. The use of nerve transfers to restore upper extremity function in cervical spinal cord injury. PM R 2018;10:1173-1184.e2.

35. Choi H, Seo KC, Kim TU, Lee SJ, Hyun JK. Repetitive transcranial magnetic stimulation enhances recovery in central cord syndrome patients. Ann Rehabil Med 2019;43:62-73.

36. Lum PS, Burgar CG, Shor PC, Majmundar M, Van der Loos M. Robot-assisted movement training compared with conventional therapy techniques for the rehabilitation of upper-limb motor function after stroke. Arch Phys Med Rehabil 2002;83:952-9.

37. Lee KW, Kim SB, Lee JH, Lee SJ, Kim JW. Effect of robot-assisted game training on upper extremity function in stroke patients. Ann Rehabil Med 2017;41:53946.

38. Hesse S, Heß A, Werner C C, Kabbert N, Buschfort R. Effect on arm function and cost of robot-assisted group therapy in subacute patients with stroke and a moderately to severely affected arm: a randomized controlled trial. Clin Rehabil 2014;28:637-47.

39. Orihuela-Espina F, Roldan GF, Sanchez-Villavicencio I, Palafox L, Leder R, Sucar LE, et al. Robot training for hand motor recovery in subacute stroke patients: a 
randomized controlled trial. J Hand Ther 2016;29:517.

40. Beekhuizen KS, Field-Fote EC. Massed practice versus massed practice with stimulation: effects on upper ex- tremity function and cortical plasticity in individuals with incomplete cervical spinal cord injury. Neurorehabil Neural Repair 2005;19:33-45. 


\section{SUPPLEMENTARY MATERIALS}

Table S1. The detailed methods of the Graded and Redefined Assessment of Strength, Sensibility, and Prehension (GRASSP)

\begin{tabular}{cc}
\hline \multicolumn{1}{c}{ Domain } & \multicolumn{1}{c}{ Description } \\
\hline Strength & Upper limb muscle strength was evaluated by the manual muscle test (MMT) and graded on \\
& a 5-point scale adapted from the Medical Research Council (MRC) scale in 10 muscles of the \\
& upper limb including anterior deltoid, biceps (C5), wrist extensors (C6), triceps and opponens \\
pollicis (C7), extensor digitorum, 3rd finger flexor and flexor pollicis longus (C8), 5th finger \\
abductor and first dorsal interossei (T1). Ten grades for each side were summed to render a total \\
strength subtest score ranging from 0-50 for each upper limb.
\end{tabular}

Data available from: http://www.grassptest.com. 
Table S2. Changes in MMT scores of key muscles and UEMSs after intervention

\begin{tabular}{|c|c|c|c|c|c|c|c|}
\hline & \multicolumn{3}{|c|}{ RT $(n=17)$} & \multicolumn{3}{|c|}{ OT $(n=13)$} & \multirow{2}{*}{$\begin{array}{c}\begin{array}{c}\text { Between group } \\
\text { differences }\end{array} \\
\text { p-value }\end{array}$} \\
\hline & Pre & Post & p-value & Pre & Post & p-value & \\
\hline $\mathrm{C} 5^{1}$ & $3.29 \pm 1.64$ & $3.76 \pm 1.85$ & $0.023^{*}$ & $4.15 \pm 1.28$ & $4.23 \pm .23$ & 0.317 & 0.074 \\
\hline $\mathrm{C} 6^{2}$ & $3.47 \pm 1.46$ & $3.88 \pm 1.36$ & 0.088 & $3.38 \pm 1.66$ & $3.84 \pm 1.46$ & $0.034^{*}$ & 0.822 \\
\hline $\mathrm{C} 7^{3}$ & $3.29 \pm 1.49$ & $3.70 \pm 1.61$ & $0.035^{*}$ & $3.76 \pm 1.42$ & $4.15 \pm 1.21$ & 0.157 & 0.638 \\
\hline $\mathrm{C} 8^{4}$ & $2.52 \pm 1.87$ & $2.64 \pm 2.02$ & 0.317 & $2.84 \pm 1.67$ & $3.07 \pm 1.89$ & 0.180 & 0.532 \\
\hline $\mathrm{T}^{5}$ & $2.00 \pm 1.65$ & $2.17 \pm 1.77$ & 0.257 & $2.15 \pm 1.46$ & $2.53 \pm 1.56$ & $0.025^{*}$ & 0.202 \\
\hline Total (UEMS) & $14.59 \pm 6.49$ & $16.18 \pm 7.25$ & $0.018^{*}$ & $16.31 \pm 6.83$ & $17.85 \pm 6.81$ & $0.010^{*}$ & 0.966 \\
\hline
\end{tabular}

Values are presented as mean \pm standard deviation.

MMT, manual muscle test; UEMS, upper extremity motor score; RT, combining upper limb robotic therapy with conventional occupational therapy; OT, only conventional occupational therapy; $\mathrm{C}^{1}$, elbow flexion; $\mathrm{C}^{2}$, wrist extension; $\mathrm{C}^{3}$, elbow extension; $\mathrm{C}^{4}$, 3rd finger flexion; $\mathrm{Tl}^{5}$, 5 th finger abduction; and the superscripts indicate key muscle function of corresponding level.

${ }^{*} \mathrm{p}<0.05$ by Wilcoxon signed-rank test. 